senkte er LDL um 36\% und ließ das HDL um 138\% hochschnellen (von 40,4 auf 102 mg/ dl nach 76 Wochen), statistisch signifikante Ergebnisse.

Am Kapitel der CETP-Hemmer wird nun mit großem Eifer weitergeschrieben, sowohl die Grundlagen- als auch die klinische Forschung ist gefordert, nicht zuletzt auch durch den Abbruch der AIM-HIGH-Studie in den USA, in der sich die "HDL-Hoffnung" retardiertes Niacin als nutzlos erwiesen hat.

\section{EMPHASIS-HF: Unterschätzter Aldosteron-Antagonist}

Der Aldosteron-Antagonist Eplerenon hat sich auch bei Patienten mit leichter systolischer Herzinsuffizienz (NYHA 2) als sehr wirksam erwiesen, wie EMPHASIS-HF zeigte. Eine weitere Auswertung, die Bertram Pitt, Ann Arbor/USA, in Paris vorstellte, bestätigte die guten Ergebnisse der Hauptstudie auch für die kritischen Subgruppen der Diabetiker und Niereninsuffizienten, der alten Patienten und solchen mit niedrigem Blutdruck und niedriger Auswurffraktion.
EMPHASIS-HF umfasst 2737 Patienten mit leichter Herzinsuffizienz und linksventrikulärer Ejektionsfraktion $(E F)<35 \%$. Die Patienten erhielten entweder Eplerenon oder Placebo zusätzlich zu ihrer Standardtherapie (Betablocker, ACE-Hemmer / Sartane, bei Bedarf auch Diuretika). Die Studie wurde schließlich vorzeitig abgebrochen wegen des klaren Vorteils der VerumGruppe. Zu diesem Zeitpunkt betrug die mittlere Nachbeobachtungszeit 21 Monate.

Primärer Endpunkt war die Kombination aus dem Versterben des Patienten oder einer Hospitalisierung wegen einer Dekompensation der Herzinsuffizienz. Er wurde nach einer Laufzeit von 21 Monaten von 18,2\% im Eplerenon-Arm gegenüber 25,9\% im Placebo-Arm erreicht.

Eine Subgruppe wurde, wie Pitt sagte, jedoch um sieben Monate weitergeführt unter Beibehaltung des Doppelblindstatus. Der primäre Endpunkt veränderte sich dadurch nicht, er blieb stabil bei einer relativen Reduktion von 34\%.

\section{Hospitalisationen nahmen stark ab}

Pitt wies darauf hin, dass der primäre Endpunkt nur die erste Hospitalisation zählt. Die ergänzende Analyse zeigt nun, dass auch die wiederholten Hospitalisationen stark abnahmen. Damit wurde nicht nur die Lebensqualität der Patienten verbessert sondern es wurden auch erhebliche Kosten eingespart.

Der eingeladene Diskutant, Piotr Ponikowski aus Warschau/Polen, bekräftigte die Bedeutung der verminderten Einweisungen in der Verum-Gruppe. Für ihn war diese Studie der letzte Beweis für die Evidenz, dass Aldosteronantagonisten in allen Stadien der Herzinsuffizienz, angefangen bei der linksventrikulären Dysfunktion nach Herzinfarkt (EPHESUS) bis zur schweren Insuffizienz (RALES) und natürlich auch bei den leichten bis mittelschweren Graden, wie jetzt EMPHASIS$\mathrm{HF}$ belegt hat, indiziert sind, sofern nicht eine Hyperkaliämie dagegenspricht.

\section{Chancen von Eplerenon nicht genutzt}

Derzeit würden diese Substanzklassen Europa und in den USA sträflich vernachlässigt,

\title{
Auf der großen Erfolgs-Welle
}

Erfolgsverwöhnte ESC: Der diesjährige Jahreskongress - erstmals in Paris brach alle Rekorde und hat auch die bislang führenden amerikanischen Großereignisse deutlich abgehängt. Programmdirektor Prof. Michael Böhm durfte in den offiziellen ESC-Highlights die Jubelmeldung verkünden: 32946 Teilnehmer wurden registriert, darunter auch über 750 Medienvertreter, die die Botschaften via Print, Audio, Video und Internet in aller Herren Länder verbreiteten. Aus so ziemlich allen $\mathrm{Na}$ tionen waren Kardiologen angereist, darunter große Gruppen aus Brasilien, Japan, China, und Indien.

\section{Out of Europe}

Die ESC ist längst über die angestammten europäischen Mitgliedsländer hinausgewachsen. 53 nationale kardiologische Gesellschafen haben sich bis Juni 2011 unter dem Dach der ESC vereint, von der albanischen, algerischen, armenischen über die georgische und moldavische bis zur uk- rainischen Fachgesellschaft, um nur einige herauszugreifen; die meisten waren mit einem Info-Stand auf dem Kongress vertreten. Auch die inzwischen 35 assoziierten Gesellschaften von Argentinien über Saudi-Arabien bis nach Vietnam waren präsent. Eine globale Show kardiologischer Kompetenz, eine gigangische Leistungsschau einschließlich einer Industriemesse. Es ist evident, die ESC dreht mittlerweile ein ziemlich großes Rad.

\section{Immer mehr Register}

Kongresspräsident Michel Komajda von der berühmten Klinik Pitié Salpetrière in Paris (eine ehemalige Schießpulverfabrik) stellte die Pariser Kardiologiepremiere unter das Motto: „Kongress der Register". Tatsächlich sind die Kardiologen zumindest in der westlichen Welt Registern gegenüber sehr aufgeschlossen, was auch zu einer Schwemme von Registerstudien geführt hat, die ein schlaglichtartiges Bild vom jeweiligen Versorgungs- und
Qualitätsstandard vermitteln können. Die Fülle an Registern ist inzwischen allerdings so groß geworden, dass die ESC Regulierungsbedarf erkannt hat. Mit dem „EURObservational Research Programme" sollen die Herz-KreislaufRegister quer durch Europa koordiniert werden. Außerdem werden weitere Register geplant, acht neue sind in der Planungsphase, in die über 10000 Patienten aufgenommen werden sollen. Erklärtes Hauptziel ist die Verankerung der Leitlinien im medizinischen Alltag. Ein weiteres ehrgeiziges Projekt: Auch die Forschung soll künftig grenzüberschreitend stärker vernetzt werden.

Dass ein solcher Megakongress jede Stadt vor logistische Probleme stellt, ist nachvollziehbar. Lediglich im abendlichen Stoßverkehr kam verschiedentlich Frust auf, sonst dominierte gute Stimmung, Pariser Stimmung eben.

Dr. med. Jochen Aumiller II 